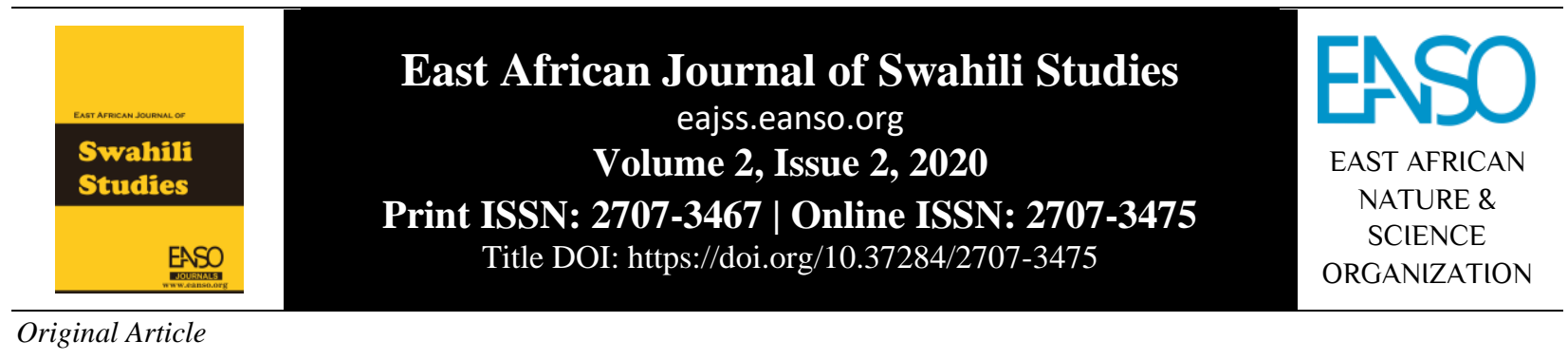

\title{
Athari za Usasa kwenye Usimulizi wa Hadithi katika Jamii ya Wamasaaba Nchini Uganda
}

\author{
Dr. Willy Wanyenya, PhD ${ }^{1}{ }^{*}$ Prof. Rocha Chimerah,Ph.D ${ }^{2}$ na Nancy Jumwa Ngowa, Ph.D ${ }^{2}$ \\ ${ }^{1}$ Makerere University Business School, S. L. P. 1337, Kampala, Uganda. \\ ${ }^{2}$ Pwani University, S. L. P. 195 - 80108, Kilifi, Kenya. \\ *Barua pepe ya mawasiliano: willywanyenya@gmail.com \\ ORCID: https://orcid.org/0000-0002-7594-8256
}

DOI ya Nakala: https://doi.org/10.37284/eajss.2.2.206

Tarehe ya Uchapishaji: IKSIRI

08 Septemba 2020 Katika makala haya, watafiti wanaeleza jinsi usimulizi wa hadithi umeathiriwa na usasa katika jamii ya Wamasaaba nchini Uganda. Wanafanya

Maneno Muhimu: hivyo kwa kulinganisha hali ilivyo sasa na jinsi ilivyokuwa kabla Wazungu hawajazuru barani afrika. Watafiti wanalinganisha miktadha ya utendaji wa Usasa, hadithi na wanatoa maelezo kuhusu mitindo mbalimbali ya utendaji. Usimulizi, Wanaeleza jinsi waafrika walivyosimulia watoto wao hadithi kabla ya Hadithi, wazungu kuja barani humu. Kwenye kazi hii, madhumuni ya utafiti ni: Sanaa, kudhihirisha athari za usasa kwenye masimulizi ya kinathari. Pili, kueleza Fasihi. stadi zilizokuwa zikitumiwa na waafrika katika usimulizi wa hadithi. Mkabala wa utafiti ulikuwa wa nyanjani. Kwa kutumia mkabala huu, watafiti walizuru nyanjani halafu wakaanza kwendeleza mahojiano na wahojiwa wao. Kabla hawajafanya hivyo, watafiti walijitambulisha kwa wahojiwa wao na kuwaambia sababu za utafiti huu. Walifanya hivyo ili kuwaondelea wasiwasi. Majibu ya wahojiwa yalijumlisha data ambazo zilihitajika na watafiti. Matokeo ya utafiti yanaonyesha kuwa usimulizi wa hadithi umefifia sana katika jamii ya Wamasaaba kutokana na usasa. Sababu ambazo zimepelekea usimulizi wa hadithi kufifia katika jamii hii pia zimeelezwa. Miongoni mwa sababu hizo ni: ulevi ambao hufanya watu kurejea nyumbani wakiwa wamelewa na kushindwa kusimulia watoto wao hadithi. Sababu nyingine ni wazazi kuthamini elimu ya kisasa na kuacha utamaduni wao wa kulea watoto ili baadaye wawe wake na waume wazuri. Wazazi kuhamasisha watoto wao wasome vitabu badala ya kuwasimulia hadithi. Nne, wazazi kuwa na lengo la kuelimisha watoto wao ili baadaye wapate kazi za afisi. Tano, jambo la kuwepo na utandawazi ambalo limepelekea watu kujumuika na kuiga tamaduni za kigeni. Upeo wa utafiti huu ulihusika na mabadiliko kwenye usimulizi wa hadithi na sababu ambazo zimepelekea utamaduni wa kusimulia hadithi kufifia katika jamii ya Wamasaaba nchini Uganda. Watafiti walitumia mbinu ya bahati nasibu kuteua sampuli za wahojiwa. 
East African Journal of Swahili Studies, Volume 2, Issue 2, 2020

Article DOI: https://doi.org/10.37284/eajss.2.2.206

APA CITATION

Wanyenya, W., Chimerah, R., \& Ngowa, N. (2020). Athari za Usasa kwenye Usimulizi wa Hadithi katika Jamii ya Wamasaaba Nchini Uganda. East African Journal of Swahili Studies, 2(2), 116-127. https://doi.org/10.37284/eajss.2.2.206

\section{CHICAGO CITATION}

Wanyenya, Willy, Rocha Chimerah, and Nancy Ngowa. 2020. "Athari za Usasa kwenye Usimulizi wa Hadithi katika Jamii ya Wamasaaba Nchini Uganda”. East African Journal of Swahili Studies 2 (2), 116-127. https://doi.org/10.37284/eajss.2.2.206.

\section{HARVARD CITATION}

Wanyenya, W., Chimerah, R. and Ngowa, N. (2020) "Athari za Usasa kwenye Usimulizi wa Hadithi katika Jamii ya Wamasaaba Nchini Uganda”, East African Journal of Swahili Studies, 2(2), pp. 116-127. doi: 10.37284/eajss.2.2.206.

\section{IEEE CITATION}

W. Wanyenya, R. Chimerah, and N. Ngowa, "Athari za Usasa kwenye Usimulizi wa Hadithi katika Jamii ya Wamasaaba Nchini Uganda”, EAJSS, vol. 2, no. 2, pp. 116-127, Sep. 2020.

\section{MLA CITATION}

Wanyenya, Willy, Rocha Chimerah, and Nancy Ngowa. "Athari za Usasa kwenye Usimulizi wa Hadithi katika Jamii ya Wamasaaba Nchini Uganda”. East African Journal of Swahili Studies, Vol. 2, no. 2, September 2020, pp. 116-127, doi:10.37284/eajss.2.2.206.

\section{UTANGULIZI}

Neno 'usasa' hurejelea mabadiliko katika jamii. Mambo ya kiutamaduni yanaachwa huku jamii zikiwa zinashika mambo mapya na mageni. Usasa umeleta mambo mengi sana katika jamii zetu. Baadhi ya mambo hayo ni mazuri na mengine ni mabaya. Watu wengi wameacha mambo yao ya kiutamaduni kutokana na usasa. Kabla ya wakoloni kuzuru barani humu, Waafrika walikuwa wakitekeleza mambo yao kulingana na tamaduni zao. Kulikuwa na jinsi walivyovaa mavazi, walivyoandaa chakula, jinsi walivyolea watoto na jinsi walivyojitawala. Baada ya wakoloni kuzuru barani humu, mambo yalianza kubadilika. Waafrika wakaanza kutekeleza mambo ya Kizungu hata bila kujiuliza kama ni mazuri au ni mabaya. Mambo mengi ya kiutamaduni yametoweka katika jamii zetu. Kwa hivyo, katika makala haya, watafiti wanadhamiria kueleza jinsi usimulizi wa hadithi umeathiriwa kutokana na usasa katika jamii ya Wamasaaba nchini Uganda. Hii ni kwa sababu utanzu huu wa fasihi huwezi ukanusurika athari za usasa kama nyanja zingine zimeathirika.

\section{SUALA LA UTAFITI}

Swala la utafiti katika kazi hii ni kuwa kwa nini usasa umepelekea utamaduni wa kusimulia hadithi kufifia katika jamii ya Wamasaaba ilhali umepelekea utamaduni huo kuimarika katika jamii za kimataifa? Kwa mujibu wa watafiti, mchango wa usasa kwenye utamaduni wa kusimulia hadithi umezua maoni mbalimbali kutoka kwa wasomi tofautitofauti yanayokinzana. Baadhi ya wasomi hao wanaona kuwa usasa umechangia pakubwa sana uimarikaji wa hadithi katika jamii na wengine wanaona kuwa usasa ndio umechangia ufifiaji wa hadithi katika jamii mbalimbali. Kwa hiyo, utafiti huu ulidhamiria kuziba pengo ambalo linasababishwa na maelezo kinzani ya wasomi tofautitofauti kuhusu mchango wa usasa katika usimulizi wa hadithi, baadhi yao wakisema kuwa usasa umepelekea usimulizi wa hadithi kuimarika katika jamii huku wengine wakisema kuwa usasa umepelekea usimulizi wa hadithi kufifia.

\section{MKABALA WA UTAFITI}

Katika kazi hii, mkabala wa utafiti ulikuwa wa nyanjani. Watafiti walitumia mkabala huu kwa sababu ulikuwa njia ya pekee ambayo ingewaleta pamoja na wazee wenye rundo la hadithi. Kwa kutumia mkabala huu, watafiti walizuru nyanjani halafu wakaendeleza mahojiano na wahojiwa wao. Wahojiwa waliteuliwa kwa kutumia mbinu ya bahati nasibu. Watafiti walitumia mbinu hii ili kuwapa watu wote fursa ya kushiriki kama wahojiwa. Taarifa zilizokusanywa zilijumlisha maelezo kuhusu athari za usasa kwenye usimulizi wa hadithi katika jamii ya Wamasaaba. Ili kufanya kazi iwe rahisi, watafiti hawakukusanya data kutoka eneo zima la Wamasaaba. Badala yake walilenga kata moja tu na wakakusanya data huko. Kata yenyewe ilikuwa ya Bukigai. Data 
zilizokusanywa kule Bukigai zilitoa picha ya eneo zima la Wamasaaba.

\section{MAPITIO YA MAANDISHI}

Kwa mujibu wa Porter (2005), sanaa ya kale ya kusimulia hadithi sasa inashughulikiwa upya kama njia ya mawasiliano ijulikanayo kama usimulizi wa hadithi kidijitali. Kwa kutumia njia hii mpya, tunaona vifaa vya kielektroniki vikitumiwa. Vifaa hivi vinajumlisha kanda za video, $C D$, fulashi, memori kadi, vinyonyi na santuri. Porter (2005) anasema hadithi zimekuwepo tangu kuumbwa kwa binadamu na ni muhimu sana katika kuridhisha nafsi zetu kuliko mambo mengine yote.

Kulingana na maelezo ya Porter (2005), sanaa ya kale ya kusimulia hadithi sasa inashughulikiwa upya kama njia ya mawasiliano. Njia hiyo inajulikana kama usimulizi wa hadithi kidijitali. Njia hii mpya huhusisha matumizi ya kanda za video, $\mathrm{CD}$, fulashi, memori kadi, vinyonyi na santuri katika usimulizi wa hadithi. Hii inaonyesha kuwa usasa umeathiri pakubwa sana nyanja ya kusimulia hadithi. Ufafanuzi huu wa Porter (2005) unaonyesha kuwa usasa umeathiri njia mbalimbali za mawasiliano. Ikizingatiwa kuwa usimulizi wa hadithi ni njia mojawapo ya mawasiliano, inamaanisha kwamba utanzu huu pia umeathiriwa na ukuaji wa teknolojia. Hata hivyo, hatuelewi jinsi usimulizi wa hadithi umeathiriwa na teknolojia katika jamii mbalimbali. Jambo hili liliacha pengo na kupelekea utafiti huu kuendelezwa.

Kwa mujibu wa Kervin, McMahon, O'Shea na Harwood (2017), usimulizi wa hadithi kidijitali huunganisha dunia mbili; dunia mpya itumiayo video, picha, na sanaa na dunia ya kale ya kusimulia hadithi kwa kutumia mdomo. Hii inamaanisha kuwa nafasi ya dunia ya kale yenye kusimulia hadithi kwa mdomo inachukuliwa na dunia ya sasa itumiayo teknolojia. Kwa hiyo si jambo la kushangaza tukiona usimulizi wa hadithi kwa mdomo ukififia katika baadhi ya jamii. Kulingana na maelezo ya Kervin, McMahon, O'Shea na Harwood (2017), yafuatayo ni mabadiliko ambayo yamesababishwa katika hadithi na teknolojia: Nafasi ya utamaduni wa kusimulia hadithi kwa mdomo imechukuliwa na teknolojia. Badala ya kutumia mdomo, hadithi zinasimuliwa kwa kutumia video, picha na runinga.
Mtazamo huu wa Kervin, McMahon, O'Shea na Harwood (2017) unadhihirisha kuwa ufifiaji wa utamaduni wa kusimulia hadithi kwa mdomo katika jamii mbalimbali umetokana na ukuaji wa teknolojia. Hii ni kwa sababu teknolojia imechukua nafasi ya mdomo. Badala ya watu kusimulia hadithi kwa mdomo, hadithi sasa husimuliwa kidijitali. Lakini hatuelewi kama jamii zote zinazoshuhudia ufifiaji wa utamaduni wa kusimulia hadithi kwa mdomo mkiwemo ile ya Wamasaaba, zimekumbatia matumizi ya teknolojia. Jambo hili liliacha mwanya ambao umezibwa katika kazi hii ya utafiti.

Meadow (2003) anasema kuwa usimulizi wa hadithi kidijitali ni kutumia vifaa vya tarakilishi kusimulia hadithi. Anasema kuna maneno mengine chungu nzima yanayoeleza jambo hili. Lakini, kwa jumla, yote hurejelea sanaa ya kusimulia hadithi kwa kutumia vifaa mbalimbali vya kiteknolojia. Vifaa hivi ni: fulashi, memori kadi, santuri, CD, mikanda na kadhalika. Anasema leo hii ni vigumu kupata watu wakisimulia hadithi kwa mdomo. Mawaidha haya ya Meadow (2003) yanatoa picha inayoonyesha kuwa utamaduni wa kale wa kusimulia hadithi kwa mdomo umepoteza nafasi yake kutokana na ukuaji wa teknolojia. Hii inamaanisha kuwa jamii mbalimbali zimekumbatia usimulizi wa hadithi kwa kutumia teknolojia.

Mawazo haya ya Meadow (2003) yanaashiria namna ambavyo usimulizi wa hadithi umeathiriwa na usasa. Kwa mfano, hadithi kusimuliwa kwa njia ya kidijitali, matumizi ya vyombo vya kisasa kama vile filamu, video, CD, fulashi na memori kadi. Matumizi ya vifaa hivi vya kielektroniki yanaonyesha kinaganaga athari ambazo zimesababishwa na usasa katika nyanja ya kusimulia hadithi.

Hata hivyo, swali ni je, jamii ya Wamasaaba imefikia kiwango hiki cha kusimulia hadithi? Je, Wamasaaba hutumia vifaa vya kielektroniki katika usimulizi wao wa hadithi? Bila shaka, maswali haya yanadhihirisha mwanya unaostahili kuzibwa. Kwa hiyo, utafutaji wa majibu kwa maswali haya ulimpelekea msomi huyu kuendeleza utafiti huu akiwa na lengo la kutaka kuziba pengo lililopo.

Kwa mujibu wa Ndalichako (2017), athari ya sayansi na teknolojia inajitokeza pia katika utanzu wa kusimulia hadithi. Wanazuoni wengi 
wanaelekea kukubaliana kwamba, kwa vile mwanadamu si kisiwa, huingiliana na binadamu toka pande mbalimbali za dunia. Kwa hiyo, hapana shaka kuwa gunduzi zinazofanywa na binadamu huyu katika uga wa kiteknolojia zinaathiri kwa kiasi kikubwa mfumo wa maisha ya binadamu huyu kwa jumla. Ndalichako (2017) anasema kuwa uwasilishaji wa hadithi enzi za kileo, umeathiriwa zaidi na sayansi na teknolojia. Vyombo mbalimbali vilivyogunduliwa vimekuwa vikitumika kutongolea hadithi na hivyo kuchangia uhai wa fasihi simulizi kuondoka katika uwasilishaji wake.

Winhurst (2016) anasema usimulizi wa hadithi kidijitali hufuata mtindo wa jadi wa kusimulia hadithi bali huingiza teknolojia inayotoa picha, sauti na nyimbo bila kutumia binadamu halisi. Anasema badala ya kuhifadhi hadithi kichwani, hadithi sasa huwa zikihifadhiwa kielektroniki kwa kutumia vifaa kama vile $C D$, fulashi na santuri. Hadithi hizo pia huwasilishwa kiteknolojia kwa kutumia runinga, kompyuta, intaneti na fesibuku. Madai haya ya Winhurst (2016) yanaonyesha kuwa badala ya usimulizi wa hadithi kutekelezwa kwa mdomo, kwa sasa, hadithi hushughulikiwa upya kwa kutumia njia za kisasa. Kama anavyosema Winhurst (2016), hadithi huwa zinasimuliwa kwa kutumia teknolojia inayotoa picha, sauti na nyimbo.

Mawazo haya ya Winhurst (2016) yalikuwa muhimu sana kwa mtafiti kwa sababu yalimwekea msingi wa kuendeleza utafiti wake. Hii ni kwa sababu katika uchunguzi wake, mtafiti alikuwa akijaribu kuthibitisha kama usimulizi wa hadithi katika jamii ya Wamasaaba umefikia kiwango hiki, yaani matumizi ya $\mathrm{CD}$, fulashi, memori kadi, runinga na video. Hata hivyo, mtafiti hakupata maelezo yoyote ya kuthibitisha kuwa usimulizi wa hadithi katika jamii ya Wamasaaba hutekelezwa kwa njia hizi za kisasa. Jambo hili liliwapelekea watafiti kuendeleza utafiti huu ili aelezee jinsi utamaduni wa kusimulia hadithi ulivyo katika jamii hii.

Cracium na Bunoiu (2016) wanasema kuwa hata kama hadithi zinazosimuliwa na walimu zinaweza kufanya wanafunzi wapendelee masomo ya sayansi na kupata motisha ya kusoma, ni muhimu sana wanafunzi kubuni hadithi zao wenyewe wanazoziwasilisha kidijitali. Cracium na Bunoiu
(2016) wanasema jambo hili huwezesha wanafunzi kukuza stadi kama utafiti na kuandika.

Matamshi haya ya Craciumna na Bunoiu (2016) yanaashiria kuwa hadithi zinazosimuliwa kidijitali huboresha kusoma kwa wanafunzi. Hii inamaanisha kuwa usimulizi wa hadithi katika jamii za kimataifa hutekelezwa kiteknolojia. Walimu huwa wakitumia vifaa vya kielektroniki kama vile fulashi, CD, memori kadi na santuri wakati wa kufundisha. Hata hivyo, hatuelewi kama jamii mbalimbali hasa zile za Kiafrika zimekumbatia mbinu hizi mpya za kufundishia wanafunzi shuleni. Hatuelewi ikiwa barani humu walimu wetu huwa wakitumia vyombo vya kusimulia hadithi madarasani. Hata hatujui kama walimu wetu huwa wakitumia usimulizi wa hadithi kama mbinu mojawapo ya kufundishia wanafunzi shuleni. Ukosefu wa maelezo hayo ulisababisha mwanya ambao ulipelekea utafiti huu kuendelezwa.

Yamac (2016) anasema kuwa usimulizi wa hadithi kidijitali hukuza si tu stadi za jadi kama kusoma, kuandika na kuongea bali pia stadi za kisasa ambazo zinahitajika sana katika mazingira ya mawasiliano. Anasema badala ya kuhifadhi hadithi kichwani kama ilivyokuwa zamani, hadithi sasa huwa zikihifadhiwa kielektroniki kwa kutumia vifaa kama vile $C D$, fulashi na santuri. Hadithi hizo pia huwasilishwa kiteknolojia kwa kutumia runinga, kompyuta, intaneti na fesibuku.

Maneno ya Yamac (2016) yanadhihirisha kwamba kuna stadi nyingi sana ambazo huwa tunazikosa kutokana na kutosimulia hadithi kidijitali. Hii inamaanisha kuwa ili tupate stadi hizo mbalimbali, tunastahili kusimulia hadithi zetu kidijitali. Swali ni je, jamii mbalimbali kote ulimwenguni zina uwezo wa kuendeleza usimulizi wa hadithi kidijitali? Hapa tunaona pengo linajitokeza ambalo bila shaka lilimchochea mtafiti na kupelekea utafiti huu kuandaliwa.

Mooney na Prins (2015) anasema kuwa kwa kutumia teknolojia ya kisasa, usimulizi wa hadithi kidijitali unawapa watu njia mbadala za kufanya ulimwengu uwe na maana. Hii ni kwa sababu watu hushirikiana katika usimulizi wa hadithi kote ulimwenguni kwa kutumia mitandao ya intaneti kama vile tovuti, fesibuku na twita. Mooney na Prins (2015) anasema kuwa matumizi ya intaneti yamewezesha watu kote ulimwenguni kugawana 
mambo mbalimbali kupitia hadithi. Hii inamaanisha kwamba usasa umeathiri pakubwa sana usimulizi wa hadithi kutokana na matumizi ya vyombo vya kisasa kama vile runinga, video, $\mathrm{CD}$, fulashi, memori kadi na vifaa vingine vingi vya kisasa.

Mawaidha ya Mooney na Prins (2015)yanaonyesha kuwa haya ni mambo yanayotekelezwa katika jamii zilizoendelea kiteknolojia na kiuchumi. Kulingana na mawazo ya msomi huyu, haya ni mambo ambayo hayawezekani kwa jamii zilizochanga na maskini kama hizi zetu za Kiafrika. Jamii kama hizo hushindwa kuwapa wananchi wao huduma muhimu kama vile matibabu, mavazi, malazi mema na chakula. Kwa jamii kama hizo, huenda ikawa vigumu kuwekeza katika teknolojia ya kusimulia hadithi. Jambo hili halijaelezwa bayana na hivyo kuacha mwanya ambao uliwapelekea watafiti kuendeleza utafiti huu.

Juppi (2015) anasema kuwa usimulizi wa hadithi kidijitali hukuza stadi maalumu za mawasiliano. Kwa mujibu wa Juppi, nyakati hizi, hadithi husimuliwa kwa kutumia teknolojia kama vile CD, fulashi na santuri. Hadithi hizo pia huwasilishwa kiteknolojia kwa kutumia runinga, kompyuta, intaneti na fesibuku. Maneno haya ya Juppi (2015) yanaonyesha jinsi usasa umeathiri usimulizi wa hadithi. Ikizingatiwa kuwa teknolojia huja na gharama kubwa, kuna uwezekano wa baadhi ya jamii kushindwa kuendeleza usimulizi wa hadithi kwa njia hii, jambo ambalo liliwapelekea watafiti kuandaa utafiti huu ili aelezee jinsi hali ilivyo katika jamii ya Wamasaaba.

Kwa mujibu wa Smeda, Dakich na Sharda (2014), usimulizi wa hadithi kidijitali huboresha mazingira ya kufunzia watoto. Kulingana na Smeda, Dakich na Sharda (2014), usimulizi wa hadithi kidijitali huhusisha wanafunzi kikamilifu na huleta matokeo mazuri kwao. Smeda, Dakich na Sharda (2014) wanasema kuwa kuanzia nyakati za kale hadi leo, usimulizi wa hadithi umetumiwa kama kifaa cha kufunzia watoto na kupitisha maarifa kutoka kizazi kimoja hadi kingine. Smeda, Dakich na Sharda wanaendelea kusema kuwa katika miaka ya hivi karibuni, mabadiliko makubwa sana yameshuhudiwa katika michakato ya kubuni hadithi na vyombo mbalimbali vya kupitisha ujumbe kwa hadhira.
Smeda, Dakich na Sharda (2014) wanaendelea kusema kuwa hadi leo, watu wanaendelea kusimulia hadithi kwa kutumia vyombo vipya vya kidijitali kama vile $\mathrm{CD}$, fulashi, memori kadi na kadhalika. Smeda, Dakich na Sharda (2014) wanasema ukuaji wa teknolojia umepelekea vifaa vya kidijitali kutumiwa madarasani. Vifaa hivi huwezesha wanafunzi na walimu wao kubuni hadithi zinazosimuliwa kidijitali. Halikadhalika, usimulizi wa hadithi kidijitali huwezesha watoto kukuza ubunifu wao na kutatua matatizo yanayowakabili.

Mawazo ya Smeda, Dakich na Sharda (2014) yanaweka bayana jinsi usasa umechangia maendeleo kwenye usimulizi wa hadithi katika jamii za kimataifa. Kwa mfano, hadithi kutumiwa katika ufundishaji shuleni kwa njia ya kidijitali na hadithi kuhifadhiwa kwenye $\mathrm{CD}$, fulashi, memori kadi santuri, vinyonyi na mikanda ya kunasia sauti. Kwa bahati mbaya, haijaelezwa kinaganaga kama jamii mbalimbali ulimwenguni zimefikia kiwango hiki. Jambo hili liliacha mwanya ambao watafiti wameuziba katika utafiti huu.

Kwa mujibu wa Cueva et al. (2015), usimulizi wa hadithi kidijitali husaidia katika kuelimisha jamii kuhusu magonjwa hatari na namna ya kujilinda. Cueva et al. (2015) anasema kuwa miongoni mwa Walaskan, usimulizi wa hadithi kidijitali hutumiwa katika kueneza taarifa kuhusu kinga za magonjwa hatari kama vile ukimwi na saratani. Anasema badala ya kuhifadhi hadithi kichwani kama ilivyokuwa zamani, hadithi sasa huwa zikihifadhiwa kielektroniki kwa kutumia vifaa kama vile $C D$, fulashi na santuri. Hadithi hizo pia huwasilishwa kiteknolojia kwa kutumia runinga, kompyuta, intaneti na fesibuku.

Mtazamo huu wa Cueva et al. (2015) unabainisha kuwa teknolojia imepelekea usimulizi wa hadithi kuimarika katika jamii. Kwa mujibu wa msomi huyu, hadithi husimuliwa kwa kutumia teknolojia. Hadithi zinazosimuliwa huelimisha jamii kuhusu mambo mbalimbali. Haya yote ni mambo mazuri na yanayostahili kutekelezwa na kila jamii. Lakini swali ni je, jamii maskini zina uwezo wa kufanya hivyo? Je, jamii zenye maafisa wanaong'ang'ania kuiba pesa zinazotolewa na serikali kwa ajili ya miradi ya maendeleo zinaweza kushughulikia usimulizi wa hadithi kwa njia hizi za kisasa na 
zinazohitaji pesa? Maswali haya yanadhihirisha pengo ambalo liliangaziwa katika utafiti huu.

Robin (2013) anasema kuwa usimulizi wa hadithi kidijitali ni njia ya kisasa ambayo inatumiwa na walimu shuleni kufundishia na huwa inahusisha wanafunzi kikamilifu. Anasema wakati wa kufundisha, walimu huwa wakitumia hadithi ambazo zimehifadhiwa kielektroniki kwa kutumia vifaa kama vile $C D$, fulashi na santuri. Anasema walimu huwasilisha hadithi mbalimbali kwa wanafunzi wao kiteknolojia kwa kutumia runinga, kompyuta, intaneti na fesibuku.

Mawazo haya ya Robin (2013) yanaweka wazi namna ambavyo usasa umeathiri usimulizi wa hadithi katika jamii za kimataifa. Kwa mfano, hadithi kutumiwa katika ufundishaji shuleni kwa njia ya kidijitali. Vyombo vya kielektroniki hutumiwa katika usimulizi wa hadithi darasani. Haya yote ni mambo mema yanayostahili kutekelezwa na walimu. Hata hivyo, hatuelewi kama jamii zote hapa ulimwenguni zina uwezo wa kuendeleza usimulizi wa hadithi madarasani kidijitali. Jambo hili linaacha mwanya ambao bila shaka umezibwa katika utafiti huu.

Foley (2013) anasema kuwa mawasiliano ya kidijitali katika madarasa ya leo yamepelekea masomo ya wanafunzi kuathirika sana. Anasema wakati wa kufundisha, walimu huwa wakitumia hadithi ambazo zimehifadhiwa kielektroniki kwa kutumia vifaa kama vile $C D$, fulashi na santuri. Anasema walimu huwasilisha hadithi mbalimbali kwa wanafunzi wao kiteknolojia kwa kutumia runinga, kompyuta, intaneti na fesibuku. Hii inamaanisha kuwa wanafunzi ambao hawana ujuzi wa kutumia teknolojia huwa wakitatizwa katika masomo yao.

Mawazo haya ya Foley (2013) vile vile yanadhihirisha namna ambavyo teknolojia imeathiri usimulizi wa hadithi katika jamii za kimataifa. Katika jamii hizo, hadithi kutumiwa katika ufundishaji shuleni kwa njia ya kidijitali. Walimu huwasilisha hadithi mbalimbali kwa wanafunzi wao kiteknolojia kwa kutumia runinga, kompyuta, intaneti na fesibuku. Lakini hatuelewi kama jamii ya Wamasaaba pia imechukua hatua kama hii. Hata hatujui kama jamii hii ina uwezo wa kuendeleza usimulizi wa hadithi kwa hizi za kisasa, ikizingatiwa kuwa kuna gharama. Ukosefu wa taarifa hizi uliacha mwanya ambao watafiti wameuziba katika utafiti huu.

\section{MATOKEO YA UTAFITI}

Katika Jedwali 1, majibu mia moja na mawili (102) kwa jumla ya majibu mia moja na ishirini (120) yakiwakilisha asilimia themanini na tano (85\%) yanadhihirisha kwamba matumizi ya pesa yamechangia kupungua kwa hadithi katika jamii ya Wamasaaba. Wahojiwa ambao walikutana na watafiti ana kwa ana walisema kwamba siku hizi, watu huwa wakishughulika na utafutaji wa pesa badala ya kufikiria jambo la kusimulia watoto wao hadithi. Saa za jioni badala ya kusimulia watoto hadithi, wao huwa wanapiga hesabu za pesa ambazo wamepata na kupanga kazi ambazo watazifanya siku itakayofuata.

\section{Jedwali 1: Maoni Mbalimbali ya Wahojiwa}

\begin{tabular}{|c|c|c|c|c|c|c|c|c|c|}
\hline Swali & $\mathbf{X}$ & $\%$ & $\mathbf{Y}$ & $\%$ & $\mathbf{N}$ & $\%$ & $\mathbf{M}$ & $\%$ & O \\
\hline $\begin{array}{l}\text { Je, unafikiri kuwepo kwa mfumo wa uchumi wa } \\
\text { kifedha umepelekea utamaduni wa kusimulia hadithi } \\
\text { kupungua katika jamii hii? }\end{array}$ & 102 & 85 & 14 & 12 & 03 & 03 & 01 & 01 & 120 \\
\hline $\begin{array}{l}\text { Je, unafikiri ukuaji wa teknolojia umepelekea } \\
\text { utamaduni wa kusimulia hadithi kupungua katika } \\
\text { jamii? }\end{array}$ & 85 & 71 & 18 & 15 & 13 & 11 & 04 & 3.3 & 120 \\
\hline $\begin{array}{l}\text { Je, unafikiri watoto kuishi shuleni umepelekea } \\
\text { utamaduni wa kusimulia hadithi kupungua katika } \\
\text { jamii hii? }\end{array}$ & 83 & 69.2 & 22 & 18.3 & 13 & 11 & 02 & 02 & 120 \\
\hline
\end{tabular}




\begin{tabular}{|c|c|c|c|c|c|c|c|c|c|}
\hline Swali & $\mathbf{X}$ & $\%$ & $\mathbf{Y}$ & $\%$ & $\mathbf{N}$ & $\%$ & $\mathbf{M}$ & $\%$ & $\mathbf{O}$ \\
\hline $\begin{array}{l}\text { Je, unafikiri watoto kusoma vitabu vyao na kufanya } \\
\text { kazi za ziada wanazopewa shuleni na walimu wao } \\
\text { umepelekea hadithi kupungua katika jamii hii? }\end{array}$ & 90 & 75 & 20 & 17 & 08 & 07 & 02 & 02 & 120 \\
\hline $\begin{array}{l}\text { Je, unadhani kuwepo kwa dini mpya na ibada } \\
\text { kumepelekea usimulizi wa hadithi kupungua katika } \\
\text { jamii? }\end{array}$ & 82 & 68.3 & 24 & 20 & 13 & 11 & 01 & 01 & 120 \\
\hline
\end{tabular}

X-Wanaokubali, Y- Wasiokubali, $N$ - Wasiojua, $M$ - Wasiokuwa na uhakika.

Wahojiwa walisema pia kuwa matumizi ya pesa yamepelekea watu kuwa walevi. Kwa hiyo, wakirudi nyumbani kama wamelewa, wao hupanda vitanda vyao na kuanza kulala bila kufikiria jambo la kusimulia watoto wao hadithi. Ulevi pia husababisha vifo kwa watu na hivyo jamii hupoteza wasimulizi mashuhuri wa hadithi. Matumizi ya pesa yamefanya watu wengine kuwa na tabia ya wizi. Kwa hiyo, badala ya kufikiria jambo la kusimulia watoto hadithi, wao wanafikiria juu ya kuenda kuiba.

Hali kadhalika, wahojiwa walisema kuwa jambo la uhamiaji pia limepelekea Wamasaaba kuishi na watu wa makabila mengine. Kwa hiyo, wahamiaji huwa wakizungumzia mambo tofauti kabisa ambayo hayana uhusiano na tamaduni za Wamasaaba, na hivyo kupelekea usimulizi wa hadithi kupungua. Kwa mujibu wa wazee wa Bududa, hadithi ambazo zimeathirika sana ni zile zinazozungumzia mashujaa mbalimbali na matendo yao ya ajabu, visasili na zile hadithi za historia. Hii inamaanisha kuwa ni vigumu watoto kupata taarifa kuhusu jamii yao. Watoto wasiposimuliwa hadithi huwa wanakosa mambo mengi sana kama ilivyo kwamba hadithi huhifadhi taarifa, huburudisha, hutahadharisha, huonya, huelimisha, hupunguza ukimwa na huelekeza jamii.

Majibu themanini na matano (85) kwa jumla ya majibu mia moja na ishirini (120) yakiwakilisha asilimia sabini na moja $(71 \%)$ yanaonyesha kwamba kukua kwa teknolojia kumechangia kupungua kwa usimulizi wa hadithi katika jamii ya Wamasaaba (Jedwali 1). Wahojiwa ambao walikutana na watafiti ana kwa ana waliwambia kuwa kutokana na teknolojia, siku hizi vijana na hata watu wazima huwa wakichukua muda mrefu wakitazama kandanda na mapigano kwenye runinga au kusikiliza redio badala ya kushughulika na usimulizi wa hadithi.

Wahojiwa themanini na matatu (83) yakiwakilisha asilimia sitini na tisa nukta mbili (69.2\%) yanaonyesha kwamba watoto kuishi shuleni kumechangia kupungua kwa utamaduni wa kusimulia hadithi katika jamii ya Wamasaaba (Jedwali 1). Wahojiwa ambao walikutana na watafiti ana kwa ana walisema kuwa siku hizi watoto hupelekwa shuleni kama wangali na umri mdogo sana na wanaishi huko kwa muda mrefu. Walisema jambo hili limefanya watoto wakose hadithi za kusimuliwa na wazazi wao.

Majibu tisini (90) yakiwakilisha asilimia sabini na tano $(75 \%)$ yanaonyesha kwamba watoto kusoma vitabu vyao na kufanya kazi za ziada wanazopewa shuleni na walimu wao kumechangia kupungua kwa usimulizi wa hadithi. Wahojiwa ambao walikutana na watafiti ana kwa ana walisema kwamba siku hizi, kila watoto wanapopata wakati huwa wakisoma vitabu vyao na kwamba wazazi wao wenyewe huwa wakiwahamasisha wasome vitabu badala ya kuwasimulia hadithi.

Majibu themanini na mbili (82) yakiwakilisha asilimia sitini na nane nukta tatu (68.3\%) yanaonyesha kwamba kuzuka kwa dini mpya na ibada kumechangia kupungua kwa utamaduni wa kusimulia hadithi katika jamii ya Wamasaaba. Wahojiwa ambao walikutana na watafiti ana kwa ana walisema kwamba siku hizi watu huwa wakikesha makanisani na kwa sababu hiyo, hawana wakati wa kusimulia wana wao hadithi. Wakiwa nyumbani, badala ya kuwasimulia watoto hadithi, huwa wakisoma biblia. 
East African Journal of Swahili Studies, Volume 2, Issue 2, 2020

Article DOI: https://doi.org/10.37284/eajss.2.2.206

Jedwali 2: Maoni ya Ziada Kuhusu Athari za Usasa Katika Hadithi

\begin{tabular}{|c|c|c|c|c|c|c|c|c|c|}
\hline Swali & $\mathbf{X}$ & $\%$ & $\mathbf{Y}$ & $\%$ & $\mathbf{N}$ & $\%$ & $\mathbf{M}$ & $\%$ & O \\
\hline $\begin{array}{l}\text { Je, unafikiri utandawazi umeathiri maendeleo ya } \\
\text { hadithi katika jamii? }\end{array}$ & 82 & 68.3 & 19 & 16 & 16 & 13.3 & 03 & 03 & 120 \\
\hline $\begin{array}{l}\text { Je, unafikiri watu kufanya kazi za afisi kumeathiri } \\
\text { maendeleo ya hadithi? }\end{array}$ & 81 & 68 & 23 & 19.1 & 13 & 11 & 03 & 03 & 120 \\
\hline $\begin{array}{l}\text { Je, unafikiri shughuli za mashirika yasiyo ya serikali } \\
\text { zimeathiri maendeleo ya hadithi? }\end{array}$ & 82 & 68.3 & 23 & 19.1 & 13 & 11 & 02 & 02 & 120 \\
\hline $\begin{array}{l}\text { Je, unafikiri wasomi kuona usimulizi wa hadithi } \\
\text { kama jambo la kishenzi na ambalo limepitwa na } \\
\text { wakati kumeathiri maendeleo ya hadithi? }\end{array}$ & 80 & 67 & 27 & 23 & 11 & 9.2 & 02 & 02 & 120 \\
\hline $\begin{array}{l}5 \text { Je, unafikiri lengo la wazazi kukuza watoto wapate } \\
\text { kazi bora badala ya kuwa wake na waume wazuri } \\
\text { kumeathiri maendeleo ya hadithi? }\end{array}$ & 83 & 69.2 & 24 & 20 & 10 & 8.3 & 03 & 03 & 120 \\
\hline
\end{tabular}

X-Wanaokubali Y- Wasiokubali N-Wasiojua M-Wasiokuwa na uhakika

Kwa mujibu wa jedwali la hapo juu, majibu themanini na mawili (82) yakiwakilisha asilimia sitini na nane nukta tatu $(68.3 \%)$ yanaonyesha kwamba utandawazi umepelekea utamaduni wa kusimulia hadithi kupungua katika jamii ya Wamasaaba. Wahojiwa ambao walikutana na watafiti ana kwa ana walimwambia kuwa kutokana na utandawazi, watu huwa wakiiga mila za makabila mengine na kuacha zile zao. Wahojiwa hao walisema kuwa watu huwa wakiiga mavazi ya kigeni, kufuata mila za kigeni na kutenda mambo ya kigeni.

Majibu themanini na moja (81) yakiwakilisha asilimia sitini na nane (68\%) yanaonyesha kuwa kufanya kazi za afisi kumepelekea watu wengi kuachana na utamaduni wao wa kusimulia hadithi. Wahojiwa wote ambao walikutana na watafiti ana kwa ana waliwambia kuwa watu wanaofanya kazi za afisi hawashughuliki na jambo la kusimulia hadithi. Mhojiwa mmoja kwa jina Bukanza aliwaambia watafiti kuwa watu wanaofanya kazi za afisi, saa za jioni huwa wakifikiria juu ya kazi ambazo wamezifanya na zile ambazo watazifanya siku itakayofuata.

Majibu themanini na mawili (82) yakiwakilisha asilimia sitini na nane nukta tatu (68.3\%) yanaonyesha kuwa kazi zinazofanywa na mashirika yasiyo ya kiserikali zimepelekea utamaduni wa kusimulia hadithi kutoweka katika jamii ya Wamasaaba. Wahojiwa wote ambao walikutana na watafiti ana kwa ana walisema kuwa mashirika haya huwa yakihamasisha vijana kuachana na mambo ya kimila na badala yake kushika mila ngeni.

Majibu themanini (80) yakiwakilisha asilimia sitini na saba (67\%) yanaonyesha kuwa wasomi kuona usimulizi wa hadithi kama jambo la kishenzi na ambalo limepitwa na wakati kumeathiri maendeleo ya hadithi katika jamii ya Wamasaaba. Mzee mmoja kwa jina Kuloba aliwambia watafiti kuwa wasomi kuona utamaduni wa kusimulia hadithi kama jambo la kishenzi na ambalo limepitwa na wakati kumeathiri sana usimulizi wa hadithi katika jamii ya Wamasaaba.

Majibu themanini na matatu (83) yakiwakilisha asilimia sitini na tisa nukta mbili (69.2\%) yanaonyesha kuwa wazazi kukuza watoto wao ili wapate kazi nzuri badala ya kuwa wake na waume wazuri kumeathiri usimulizi wa hadithi. Kulingana na maelezo ya wahojiwa, Watu wa siku hizi huwa wanapendelea watoto wao wasome ili waweze kupata kazi za afisini. Kwa hiyo, wazazi hufanya liwezekanalo kuhakikisha kuwa watoto wao 
wamepata elimu bora. Hivyo basi, jambo la kusimulia hadithi hutazamwa kama lisilo na maana.

\section{MJADALA WA MATOKEO}

Uwasilishaji wa matokeo ya utafiti unabainisha kuwa uchumi wa kifedha au matumizi ya pesa umechangia kupungua kwa hadithi katika jamii. Jambo hili linathibitishwa na idadi kubwa sana ya wahojiwa mia moja na wawili ambao walikubali hoja ikilinganishwa na idadi ndogo sana ya wahojiwa kumi na wanne ambao walipinga au wahojiwa watatu ambao hawakujua jibu sahihi au mhojiwa mmoja ambaye hawakuwa na uhakika.

Jambo hili halimaanishi kuwa watu waache kazi za biashara halafu wakazane na usimulizi wa hadithi. Biashara ni jambo muhimu sana katika maisha ya binadamu. Watu wengi sana hujikimu maishani kutokana na biashara. Hii inamaanisha kuwa watu kama hao wakiacha biashara zao kwa ajili ya kusimulia hadithi, hawataweza kupata pesa za kugharamia mambo mbalimbali. Kwa mfano hawataweza kununulia aila zao chakula, mavazi au hawataweza kulipia watoto wao karo za shule na matibabu kila wanapokuwa wagonjwa.

Kwa hiyo, jambo linalohitajika hapa ni kuweka njia mbadala za kuendeleza usimulizi wa hadithi vile jamii zingine zimefanya, kwa mfano kusimulia hadithi kidijitali na kutumia vyombo mbalimbali vya kielektroniki kama vile $\mathrm{CD}$, fulashi, memori kadi na kadhalika.

Uchanganuzi wa data pia unaonyesha kuwa ufifiaji wa utamaduni wa kusimulia hadithi katika jamii ya Wamasaaba umetokana na watoto kuishi shuleni. Jambo hili linathibitishwa na idadi kubwa sana ya wahojiwa themanini na watatu ambao walikubali hoja ikilinganishwa na idadi ndogo sana ya wahojiwa ishirini na wawili ambao walipinga au wahojiwa kumi na watatu ambao hawakujua jibu sahihi au wahojiwa wawili ambao hawakuwa na uhakika. Hata hivyo, jambo hili halimaanishi kuwa watoto waondolewe katika shule za mabweni na kuwekwa katika zile za kutwa kwa ajili ya kurudi nyumbani na kusimuliwa hadithi saa za usiku.

Katika ulimwengu wa sasa, elimu ni jambo muhimu sana kwa binadamu. Bila elimu maisha huwa magumu sana. Kwa hiyo, kila mzazi anajaribu iwezekanavyo kuwapa watoto wake elimu bora na ya kutosha. Kwa sababu bila mtu kuwa na elimu bora na ya kutosha, hakuna mafanikio ambayo anaweza kuyapata katika ulimwengu huu wa utandawazi. Hii ndiyo sababu wazazi wanatafuta shule ambazo wanafikiria zitawapa wana wao elimu inayofaa. Nyingi ya shule hizo ni za watoto kuishi palepale na watoto hulazimika kufanya hivyo. Kwa hiyo, jambo linalohitajika hapa ni kuweka njia mbadala za kushirikisha watoto hao katika usimulizi wa hadithi, kwa mfano walimu kufunza kwa kutumia hadithi, watoto kusimuliwa hadithi kidijitali wakiwa shuleni na kutumia vifaa vya kielektroniki kama vile $C D$, fulashi, memori kadi na kadhalika kama mataifa yaliyoendelea yanavyofanya.

Uchanganuzi wa data vilevile unaonyesha kuwa watoto kusoma vitabu vyao na kufanya kazi za ziada wanazopewa shuleni na walimu wao kumeathiri sana usimulizi wa hadithi katika jamii ya Wamasaaba. Jambo hili linathibitishwa na idadi kubwa sana ya wahojiwa tisini ambao walikubali hoja ikilinganishwa na idadi ndogo ya wahojiwa ishirini ambao walipinga au wahojiwa wanane ambao hawakujua jibu sahihi au wahojiwa wawili ambao hawakuwa na uhakika.

Hata hivyo, jambo hili halimaanishi kuwa watoto waache kusoma vitabu kwa ajili ya kusimuliwa hadithi. Kwa sababu usomaji wa vitabu ni jambo la kimsingi kwa msomi yeyote. Watoto wanaoendelea vizuri shuleni huwa wakisoma vitabu vyao kila wanapopata wakati hasa saa za jioni na usiku. Hii ndiyo sababu wazazi wengi huwa wakiwahimiza watoto wao wawe wakisoma vitabu badala ya kuwasimulia hadithi. Hata hivyo, utafiti huu umeonyesha kuwa usimulizi wa hadithi pia ni muhimu kwa sababu huwapa watoto taarifa kuhusu mambo ya kiutamaduni. Kwa hiyo, jambo linalohitajika hapa ni kubuni njia mbadala za kusimulia watoto hadithi ili wapate kunufaika.

Uchanganuzi wa data katika sura ya nne vilevile unaonyesha kuwa kuzuka kwa dini mpya na ibada kumeathiri sana usimulizi wa hadithi katika jamii ya Wamasaaba. Jambo hili linathibitishwa na idadi kubwa ya wahojiwa themanini na wawili ambao walikubali hoja ikilinganishwa na idadi ndogo ya wahojiwa ishirini na wanne ambao walipinga au wahojiwa kumi na watatu ambao hawakujua jibu 
sahihi au mhojiwa mmoja ambaye hakuwa na uhakika.

Hata hivyo, jambo hili halimaanishi kuwa watu waache kuabudu Mungu kwa njia za kisasa halafu waendeleze ibada zao kwa njia za jadi. Dini hizi mpya zimepelekea watu kumwabudu Mwenyezi Mungu aliye hai na ambaye ni muumba wa kila kitu. Dini za jadi zilipelekea watu kuabudu miungu mbalimbali kama inavyojulikana katika jamii tofauti kote ulimwenguni. Kwa hiyo, jambo linalohitajika hapa ni kuingiza usimulizi wa hadithi kanisani. Makasisi na wahubiri wengine wanaweza kufanya hivyo kwa kutoa mahubiri yao kwa njia ya kusimulia hadithi kiteknolojia au kwa mdomo. Jambo hili likitendeka, basi, hata waumini watanufaika na utamaduni huu wa kusimulia hadithi utaimarika katika jamii.

Uchanganuzi wa data pia unaonyesha kuwa utandawazi umechangia kupungua kwa hadithi katika jamii ya Wamasaaba. Jambo hili linathibitishwa na idadi kubwa ya wahojiwa themanini na wawili ambao walikubali hoja ikilinganishwa na idadi ndogo ya wahojiwa kumi na tisa ambao walipinga au wahojiwa kumi na sita ambao hawakujua jibu sahihi au wahojiwa watatu ambao hawakuwa na uhakika. Kama ilivyo na athari zingine ambazo zimekwisha tajwa, jambo hili vilevile halimaanishi kuwa utandawazi uangamizwe katika jamii ya Wamasaaba. Kwa sababu utandawazi umepelekea Wamasaaba pia kufanya biashara na mataifa ya kigeni. Kwa mfano, wao ni wakulima wa kahawa na kutokana na utandawazi, kahawa hiyo huuzwa katika soko la kimataifa.

Wamasaaba hufanya hivyo kupitia mashirika mbalimbali kama vile Kawacom, Kyagalanyi na Bugisu Cooperative Union ambayo yanafanya hiyo kazi ya kusafirisha kahawa hadi soko hilo la kimataifa. Utandawazi pia umepelekea nchi mbalimbali kuwa na mifumo sawa ya utawala kwa kutegemea misingi ya demokrasia. Utandawazi umeleta watu pamoja na kufanya ulimwengu uwe kama mtaa. Hii ni kwa sababu watu huwasiliana kwa urahisi sana kwa kutumia mitandao ya intaneti, simu za rununu, barua pepe na faksi. Hii inadhihirisha wazi kuwa utandawazi hauwezi kutupiliwa mbali.
Kwa hiyo, kinachohitajika hapa ni Wamasaaba kuanza kuendeleza usimulizi wa hadithi kwa njia za kisasa kama vile kusimulia hadithi kidijitali na kutumia teknolojia inayotoa picha, sauti na muziki kama vile runinga, video na tarakilishi. Wanaweza pia kutumia vifaa mbalimbali vya kielektroniki kama vile $\mathrm{CD}$, fulashi na memori kadi.

Kulingana na data zilizochanganuliwa, watu kufanya kazi za afisini kumeathiri sana usimulizi wa hadithi katika jamii ya Wamasaaba. Jambo hili linathibitishwa na idadi kubwa ya wahojiwa themanini na mmoja ambao walikubali hoja ikilinganishwa na idadi ndogo ya wahojiwa ishirini na watatu ambao walipinga au wahojiwa kumi na watatu ambao hawakujua jibu sahihi au wahojiwa watatu ambao hawakuwa na uhakika kuhusu jibu sahihi. Hii haina maana kuwa watu waache kufanya kazi za afisini kwa ajili ya kuendeleza utamaduni wa kusimulia hadithi.

Kazi za afisini zimepelekea watu wengi kuachana na kazi ngumu na chafu za mashambani. Kutokana na mambo mazuri yanayopatikana kwa sababu ya watu kufanya kazi za afisini, watu wengi wanajaribu iwezekanavyo kupata kazi za aina hiyo. Hii ni kwa sababu watu wanaofanya kazi za aina hiyo huishi maisha mazuri na ya kupendeza. Wana uwezo wa kugharamia mambo mbalimbali kama vile kununulia familia zao chakula, mavazi, kulipa kodi za nyumba wanamoishi kama ni za kupanga na pia wanaweza kulipia wana wao karo za shule.

Kwa hiyo, kutokana na mambo haya, watu hawawezi kuacha kufanya kazi za afisini kwa ajili ya kusimulia hadithi. Kinachohitajika hapa ni kuendeleza usimulizi wa hadithi kwa njia za kisasa kama vile kutumia teknolojia inayotoa sauti picha na muziki. Teknolojia hii inajumlisha vyombo kama vile runinga, video na tarakilishi. Aidha, wanaweza kutumia vyombo vya kielektroniki kama vile $C D$, fulashi na memori kadi.

Hali kadhalika, shughuli zinazoendelezwa na mashirika yasiyo ya kiserikali pia zimeathiri sana usimulizi wa hadithi kwa sababu mashirika hayo huwa yakifunza watu mambo ya kisasa na kuacha mambo yao ya kijadi kama vile usimulizi wa hadithi. Jambo hili vilevile linathibitishwa na idadi kubwa ya wahojiwa themanini na wawili ambao walikubali hoja ikilinganishwa na idadi ndogo ya wahojiwa ishirini na watatu ambao walipinga au 
wahojiwa kumi na watatu ambao hawakujua jibu sahihi au wahojiwa wawili ambao hawakuwa na uhakika kuhusu jibu sahihi.

Hata hivyo, hii haimaanishi kuwa mashirika hayo yapigwe marufuku na yasifanye kazi zao katika jamii ya Wamasaaba. Kwa sababu mashirika haya yanafanya kazi nzuri sana kwa jamii husika. Kwa mfano, yanatoa misaada kwa watoto yatima na wale wanaotoka katika familia masikini na pia kwa waathiriwa wa miporomoko ya ardhi. Mashirika haya pia yameelimisha watoto wengi ambao wazazi wao hawajiwezi. Kwa hiyo, kulingana na shughuli hizi muhimu, mashirika hayo hayawezi kupigwa marufuku kwa ajili ya kuendeleza utamaduni wa kusimulia hadithi. Kinachohitajika hapa ni kuhimiza mashirika hayo yaelimishe jumuiya kuhusu umuhimu wa kusimulia hadithi.

Kwa mfano, kulingana na mtaala wa elimu, nchini Uganda watoto wanastahili kufunzwa katika lugha za mama kuanzia darasa la kwanza hadi la nne. Kwa hiyo, usimulizi wa hadithi unaweza kuwa njia mojawapo ya kufunzia watoto shuleni. Aidha, baada ya kuchanganua data, imebainika wazi kuwa wasomi kuona usimulizi wa hadithi kama jambo la kale na ambalo limepitwa na wakati kumeathiri usimulizi wa hadithi. Jambo hili pia linathibitishwa na idadi kubwa ya wahojiwa themanini ambao walikubali hoja ikilinganishwa na idadi ndogo ya wahojiwa ishirini na saba ambao walipinga au wahojiwa kumi na mmoja ambao hawakujua jibu sahihi au wahojiwa wawili ambao hawakuwa na uhakika kuhusu jibu sahihi.

Wazo hili limeshamiri sana miongoni mwa wasomi ambao si wa fasihi. Kwa wasomi wa fasihi, mambo ni tofauti. Wao wanaheshimu usimulizi wa hadithi na wamefanya jitihada za kuendeleza utamaduni huo. Lakini wenzao kutoka nyanja zingine za usomi, usimulizi wa hadithi si suala kwao. Wanaona hili ni jambo la kale sana na ambalo limepitwa na wakati. Fikra potovu kama hizi zimepelekea utamaduni wa kusimulia hadithi kufifia katika jamii ya Wamasaaba. Jambo hili linaweza kutatuliwa kwa kuanzisha njia mbadala zinazohusisha wasomi hao kutoka nyanja zingine katika usimulizi wa hadithi.

Hatimaye, uchanganuzi wa data katika sura ya nne unadhihirisha kuwa jambo la wazazi kukuza watoto wao ili wapate kazi nzuri badala ya kuwa wake na waume bora kumeathiri pakubwa sana usimulizi wa hadithi. Kwa sababu siku hizi wazazi wanapendelea kupeleka watoto wao shuleni ili wapate elimu ya kisasa na baadaye wapate kazi nzuri za afisini.

Hawashughuliki na kazi ya kuwalea watoto wao ili wawe wake na waume bora. Jambo hili linathibitishwa na idadi kubwa ya wahojiwa themanini na watatu ambao walikubali hoja ikilinganishwa na idadi ndogo ya wahojiwa ishirini na wanne ambao walipinga au wahojiwa kumi ambao hawakujua jibu sahihi au wahojiwa watatu ambao hawakuwa na uhakika kuhusu jibu sahihi. Hata hivyo, jambo hili halimaanishi kuwa wazazi waache kuelimisha wana wao kwa ajili ya kuwasimulia hadithi ili baadaye waweze kuwa wake na waume shupavu. Wazazi wanapaswa kuelimisha watoto wao. Kwa sababu watu wanaofanya kazi za afisini huishi maisha mazuri na yanayopendeza. Mtoto wa kike ambaye ameelimika hategemei mumewe kama yule ambaye hajaelimika.

Vilevile, mvulana ambaye ameelimika hatesi mkewe kama yule ambaye hajakwenda shuleni. Ni kweli kwamba watoto wanaokwenda shuleni hukosa mambo mengi sana ya kiutamaduni. Hata hivyo, hili si jambo la kufanya wazazi waache kuelimisha wana wao. Kinachohitajika hapa ni kuendeleza usimulizi wa hadithi kwa njia za kisasa ambazo ni kusimulia hadithi kidijitali na kutumia teknolojia inayotoa picha, sauti na muziki kama vile runinga, video na tarakilishi.

\section{HITIMISHO}

Kwa kuzingatia data ambazo zimewasilishwa na kujadiliwa katika makala haya, inabainika wazi kuwa hatua zisipochukuliwa, usimulizi wa hadithi unaendelea kutoweka katika jamii zetu. Kama ilivyo na jamii nyingi za Kiafrika, usimulizi wa hadithi ni njia ya jadi ya kuelimishia watoto. Kwa kutumia njia hii, watoto huwa wanafunzwa mambo mbalimbali kama vile kupika chakula, kulima, kuweka nyumba safi nk. Hii inamaanisha kuwa usimulizi wa hadithi una mchango mkubwa sana katika maisha ya vijana. Kwa hiyo, haifai watu kupuuza utanzu huu wa fasihi simulizi. Yafaa utanzu wa fasihi ukuzwe badala ya kuangamizwa. Kutokana na umuhimu huu wa kusimulia hadithi, viongozi katika jamii ya Wamasaaba wanastahili 
kuweka mikakati mbalimbali ya kukuza utanzu huu wa fasihi simulizi. Hata si Wamasaaba pekee yao bali jamii mbalimbali za Kiafrika zinastahili kufanya hivyo. Viongozi wa jamii mbalimbali za Kiafrika wanastahili kuweka mikakati ya kukuza utanzu huu wa fasihi simulizi. Serikali kuu za nchi mbalimbali za Kiafrika zinapaswa kuweka sera madhubuti kuhakikisha kuwa utanzu huu wa fasihi simulizi unakuzwa na kuendelezwa. Aidha, ni wajibu wa wanafasihi kuhakikisha kuwa utanzu huu wa fasihi simulizi unatafitiwa na kubainisha jinsi hali ilivyo katika Jumuiya ya Afrika Mashariki na hata bara zima la Afrika.

\section{MAREJELEO}

Crăciun, D., Crăciun, P., \& Bunoiu, M. (2016, March). Digital storytelling as a creative teaching method in Romanian science education. In AIP Conference Proceedings (Vol. 1722, No. 1, p. 310001). AIP Publishing LLC.

Cueva, M., Kuhnley, R., Revels, L., Schoenberg, N. E., \& Dignan, M. (2015). Digital storytelling: a tool for health promotion and cancer awareness in rural Alaskan communities. International Journal of Circumpolar Health, 74(1), 28781.

Fikriah, F. (2016). Using the Storytelling Technique to Improve English Speaking Skills of Primary School Students. English Education Journal, 7(1), 87-101.

Foley, L. M. (2013). Digital storytelling in primary-grade classrooms. $\mathrm{PhD}$ Thesis. Arizona State University.

Juppi, P. (2015). Using Digital Storytelling to Enhance Digital Participation-A Case Study from Tanzania. Arts Academy, Turku University Press, Turkuamkfi, Turkey.

Kervin, L., McMahon, S., O'Shea, S. E., \& Harwood, V. (2014). Digital storytelling: Capturing the stories of mentors in Australian Indigenous Mentoring Experience. University of Wollongong.

Meadows, D. (2003). Digital Storytelling: Research-Based Practice in New Media. Visual Communication, 2, 189-193.
Mooney, A., \& Prins, E. (2015). Digital Storytelling in Family Literacy Programs. Practitioner's Guide\# 5. Goodling Institute for Research in Family Literacy, Penn State College of Education.

Ndalichako, J. (2017). Fasihi Simulizi na Sayansi na Teknolojia. Dar es Salaam, TZ: Taasisi ya Uchunguzi wa Taaluma za Kiswahili, Chuo Kikuu cha Dar es Salaam.

Porter, B. (2005). The art of digital storytelling. Part I: Becoming $21^{\text {st }}$ Century Story Keepers. Revista Discovery Education

Robin, B. R. (2016). The power of digital storytelling to support teaching and learning. Digital Education Review, (30), 1729.

Smeda, N., Dakich, E., \& Sharda, N. (2014). The effectiveness of digital storytelling in the classrooms: a comprehensive study. Smart Learning Environments, 1(1), 6.

Yamac, A., \& Ulusoy, M. (2017). The effect of digital storytelling in improving the third graders' writing skills. International Electronic Journal of Elementary Education, 9(1), 59-86. 